\title{
INDICE I.2.6
}

Prefazione $\quad$ V

Criteri editoriali $\quad$ VI

Curatori IX IX

Revisori dei papiri $\quad X$

Siglorum et compendiorum explicatio XII

$\begin{array}{ll}\text { Conspectus librorum } & \text { XIV }\end{array}$

GALENUS 3

1 - P.Berol. inv. 11739A. Prolegomena di un commentario a De sectis 5

2 - P.Berol. inv. 21178. Citazione/parafrasi di De naturalibus facultatibus I 214

HERODOTUS 17

1 - P.Oxy. XVII 2087, col. II 31-33. Voce di lessico (Hdt. I 32, 8) 31

2 - P.Berol. inv. 13360. Voci di lessico (Hdt. V 116 e I 60) 34

3 - P.Oxy. I 19. Nota interlineare (diortotica?) a I 76, 3

4 - P.Amh. II 12. Aristarco, Commentario a I 171, 4?-215, 2

5 - P.Oxy. VIII 1092. Nota marginale (v.l.?) a II 162, 5

6 - P.Oxy. XIII 1619. Note marginali a III 31, 2; 54, 2; 60, 3

7 - P.Oxy. LXV 4455. Commentario a $V$ 52-55 74

8 - P.Oxy. XVII 2087, col. II 27-30, 40-41. Voci di lessico (Hdt. VI 12, V 74) 90

HESIODUS 93

1 - P.Ant. III 178. Interlinear glosses on Theogony 102

2 - P.Mich. inv. 4270. Marginal glosses (now lost) on Theogony 105

3 - P.Oxy. LXVIII 4648. Discourse on poets' knowledge of constellations, with references to Works and Days 107

4 - P.Oxy. LXVIII 4652. Glossary to Shield 124

5 - P.Oxy. XXVIII 2484. Note on Catalogue of Women fr. 30 M.-W. (20 H., 27 Most) 133

6 - PSI XV 1465 + P.Oxy. XXX 2511. Marginal notes on Catalogue of Women fr. 96 H. and fr. 212(b) M.-W. (99 H., 152 Most) 135

Schede 139

(a) P.Oxy. LXVIII $4649 \quad 139$

(b) P.Oxy. LXVIII 4650

(c) P.Ant. II $60 \quad 140$ 
(d) P.Oxy. LXVIII 4651

(e) P.Berol. inv. 17042

(f) P.Oxy. XXXVII 2822, fr. 1

HIPPOCRATES 145

1 - P.Flor. II 115. Commentario a De alimento 38-39 (Galeno?) 151

2 - P.Berol. inv. 9764. Citazione di Aph. I 15

3 - P.Ant. I 28. Titoletti marginali di Aph. I 2, 3

4 - P.Ant. III 124. Citazione di Aph. I 16

5 - P.Köln I 19. Glossa a Aph. II 21 penetrata nel testo? 163

6 - P.Ant. III 183. Note marginali a Aph. III 24, 27; IV 1, 4-5 165

7 - P.Ryl. III 530. Aph. IV 77-78, V 7, 8 (?), 9, 10 (?), 11 (?), 12-14, 16-22 con parafrasi-commento 172

8 - P.Oxy. LXXX 5232. Citazione di Aph. VI 12 e del nome di Ippocrate 184

9 - P.Stras. inv. G 26. Citazione di Art. 8, Nat. hom. 4 e Morb. I 2

10 - P.Oxy. LXXX 5231. Commentario adespoto a Epid. I 192

11 - P.Herc. 1012. Citazione di Epid. VI 5, 15; 7, 9

12 - P.Oxy. III 437, 7-11. Citazione di Iusiurandum 3, 5

13 - P.Oxy. LXXIV 4970. Menzione di Iusiurandum 205

14 - P.Stras. inv. G 26. Citazione di Morb. I 208

15 - P.Stras. inv. G 26. Citazione di Nat. hom. $4 \quad 209$

16 - P.Herc. 831, col. II 1-6. Citazione di Prog. 7 in opera di Demetrio Lacone 210

17 - P.Tebt. II 678, col. IV 6-11. Citazione di Prog. 15

in un trattato sulla prognosi 212

18 - P.Herc. 1012, coll. XVIII 10 ss., XXI 1 s., XXIII 1-8.

Citazione di Prorrh. I 1-2, Epid. VI 5, 15; 7, 9 in opera di Demetrio Lacone 215

19 - P.Tebt. III 897 (= P.Bingen 1). Altra versione o commento di Vict. II 49 ? 221

Schede 224

(a) P.Oxy. LXXX 5221 (+ P.Köln VII 311) 224

(b) P.Ryl. I 56v 225

(c) P.Schøjen inv. MS 2634/3 + P.Princ. inv. AM 15960A 226

HIPPONAX 229

1 - P.Oxy. XVIII 2174. Note marginali e interlineari 233

2 - P.Oxy. XVIII 2176. Commentario a frr. 129-131 D. =118 W.2.

Note marginali e interlineari 242

3 - P.Stras. inv. G 3a-b. Annotazioni marginali e interlineari ai frr. ${ }^{\circ} 194-{ }^{\circ} 196$ D.; ${ }^{*} 115-{ }^{*} 117 W^{2}{ }^{2}$

4 - O.Berol. inv. 12605. Voce di lessico (?) 295 
Schede

(a) P.Oxy. XXXVII 2811

(b) P.Schub. 10

302

Index papyrorum

305

Index tabularum 